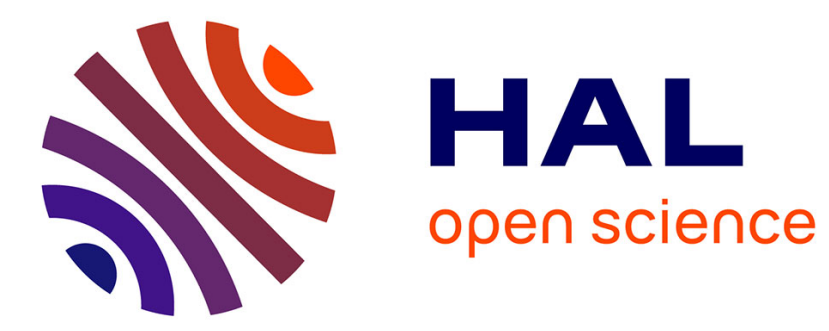

\title{
Segmentation of Mitochondria in Intracellular Space
}

\author{
Nhan Nguyen-Thanh, Tuan D. Pham, Kazuhisha Ichikawa
}

\section{To cite this version:}

Nhan Nguyen-Thanh, Tuan D. Pham, Kazuhisha Ichikawa. Segmentation of Mitochondria in Intracellular Space. 2013 IEEE Symposium on Computational Intelligence in Bioinformatics and Computational Biology (CIBCB 2013), Apr 2013, Singapour, Singapore. 10.1109/CIBCB.2013.6595412 . hal-02284801

\section{HAL Id: hal-02284801 \\ https://hal.science/hal-02284801}

Submitted on 12 Sep 2019

HAL is a multi-disciplinary open access archive for the deposit and dissemination of scientific research documents, whether they are published or not. The documents may come from teaching and research institutions in France or abroad, or from public or private research centers.
L'archive ouverte pluridisciplinaire HAL, est destinée au dépôt et à la diffusion de documents scientifiques de niveau recherche, publiés ou non, émanant des établissements d'enseignement et de recherche français ou étrangers, des laboratoires publics ou privés. 


\section{Segmentation of Mitochondria in Intracellular Space}

\author{
Nhan Nguyen-Thanh and Tuan D. Pham \\ Aizu Research Cluser for Medical Engineering and Informatics \\ Center for Advanced Information Science and Technolog \\ The University of Aizu \\ Fukushima 965-8580, Japan \\ Email: nhannt, tdpham@u-aizu.ac.jp
}

\author{
Kazuhisha Ichikawa \\ Department of Cancer Biology \\ Division of Mathematical Oncology \\ The Institute of Medical Science \\ The University of Tokyo, Tokyo 108-8639, Japan \\ Email: kichi@ims.u-tokyo.ac.jp
}

\begin{abstract}
Information of cellular organelle location and morphology is essential for cancer simulation. In order to obtain such information, the segmentation of the organelles from electronic microscopy intracellular image is crucial. In this paper, we focus on the segmentation of mitochondria organelle which is one of the most important organelles tightly related to the form of cancer. A simple three-stage strategy for mitochondrial segmentation based on exclusive and morphology properties and Gabor filter is proposed. Experimental results on focused ion beam (FIB) and scanning electron microscope (SEM) images have shown the effectiveness of proposed method.
\end{abstract}

Index Terms - cell organelles, image segmentation, image processing, mitochondria.

\section{INTRODUCTION}

A mitochondrion is one of the main organelles that can be found in most eukaryotic cells. This type of organelle concerns with the roles of energy supply, signaling and control of cellular differentiation, growth and death. It is reported that there is a strong link between the function of mitochondria and cancer. [1]. For example, the function of mitochondria of cancer cell is believed to be altered for resistance apoptosis [2], [3]. This leads the research to target on mitochondria for cancer therapy by changing mitochondrial metabolism or stimulating mitochondrial membrane permeabilization [4]. In addition, it is convinced that there are a relation of mitochondrial morphology and molecular controlling mechanisms during cell death [5], [6] and a connection of mitochondrial dynamics to key signaling cascades [7]. Therefore, obtaining information of location and morphology of mitochondria in intracellular space is very useful for cancer simulation.

Recent progresses on super-resolution electronic microscopy enable us to obtain this information through intracellular image. Some earlier methods such as ion-abrasion (IA) or serial block-face (SFB) can be combined with scanning electron microscopy (SEM), which is well-known for capturing surface characteristics and morphology, to obtain a lower resolution of both intercellular and intracellular space. Lately, the detail complex structure of the cell can be acquired with the combination of SEM and focused ion beam (FIB) technique, which increase the resolution of the scan. As a result, the integrated imaging system FIB-SEM has now been a powerful scientific instrument for the study of biological specimens and soft materials [13].
Automatically analyzing and understanding such kind of intracellular electron microscopy images are not a trivial task since the space of cellular contains a complicated system with variety of organelles possessing various shapes and sizes. There are some recent works that succeeds in segmentation of mitochondria [10]-[12] as well as some other cell organelles [8], [9]. In detail, in [12], a textural-based Gentle-Boot classifier is trained for detecting mitochondria in electron microscopy images of brain tissue. Similarly, in [11], multiple classifiers including $\mathrm{k}$-nearest neighbor algorithm ( $\mathrm{k}$ $\mathrm{NN}$ ), support vector machine (SVM) and adaptive boosting algorithm (Adaboost) are used for detecting mitochondria and other organelles of highly pigmented human melanoma (MNT-1) cell based on textural feature. These works were said to be under-utilization of image information, i.e., shape information, and were improved in [10] by considering a graph partitioning scheme incorporated with a learn shape feature method. Though the results of these research are reasonable, the huge load works for training the system could be the most difficulty for implementation.

In this paper, we propose a simple three-stage strategy for mitochondrial segmentation based on exclusive and morphological properties and Gabor filter. The using of exclusive and morphological techniques ensures a utilization of shape information for segmentation while the Gabor feature extraction enables us to utilize the texture information for detection. This combination provides a simple but reliable method for mitochondrial segmentation. The proposed method is adopted to the cancer cellular of human head and neck squamous cell carcinoma (SCC-61) images captured by FIB-SEM system.

The remainder of this paper is organized as follows. In section II, mitochondrial segmentation is described. In section III, the experiential results are provided. Finally, section V is the conclusion.

\section{MITOCHONDRIAL SEGMENTATION}

FIB-SEM intracellular images reveal a great detail structure of the cellular space which includes variety types of organelles with difference sizes, shapes, texture and brightness. The solid distribution of these organelles leads the segmentation task for intracellular images to be very complicated. Furthermore, in cases of images of abnormal cellulars such as cancer cells derived from malignant tumor, the structure and other 
characteristics of organelles tend to be sophisticated which cause the hard segmentation task to be harder.

We focus on segmentation of mitochondria, since mitochondria is one of the big organelles and play a very important role for the living of cell. The exclusive properties of segmentation task will be the major idea. In general, the mitochondria can be segmented by step by step removing other organelles. In turn, the segmentation of mitochondria will facilitate the future segmentation of the remainder organelles. The whole strategy for mitochondrial segmentation, as shown in Figure 1, includes the three following steps.

\section{A. Step 1: Coarse segmentation}

In this step, other cell organelles will be basically excluded by relevant morphological operator. Obviously, the results from this step are only limited at the raw level of determining coarse location of suspected organelles which are similar to mitochondria in aspect of morphology. First, the high frequency structure of edges and ridges of mitochondria is enhanced by high pass filtering. The filtered gray image is next changed to binary image by threshold of Otsus method [17]. The output binary image is continuously removed isolated small regions which could belong to cytoplasm, free protein or small vesicle. Next, we remove the boundaries of cell and nucleus membranes since these areas are quite large and isolated. The hardest step in coarse segmentation of mitochondria is the removal task of filament structures since these filament structures, which can originate from the endoplasmic reticulum, golgi apparatus or cytoskeleton organelles, have random shapes and stick to each other. Therefore, multiple sub-steps is required for this removing task. First, we remove any structures that are isolated and have the solidity of over $95 \%$. This operator is derived from the fact that most regions of binary mitochondria image contain empty area, in other word, have low solidity. Next, we apply a combination of the morphology operators such as opening, closing, dilating, or eroding and Canny edge detector [16] for the every large separated region. The morphology operators are objected to ellipsoid and circle structures since the tubular structure mitochrodria usually forms a circular or elliptical cross section. These operators improve the utilization of shape information for segmentation, and the edge detection strongly support for preserving edge and the ridge and valley structure of mitochondria image texture. The output of this coarse segmentation step provides us separated suspected areas which mostly contain mitochondria.

\section{B. Step 2: Detection}

The features of previous coarse segmented regions will be extracted for determining if they contain mitochondria or not. This step is the most important stage since the accuracy of whole segmentation method is mainly decided here. In this paper we consider Gabor filter for feature extraction and detection. The reason for choosing this type of feature extraction is due to the fact that Gabor filtering is well-known as an invariant and effective method for extracting texture features

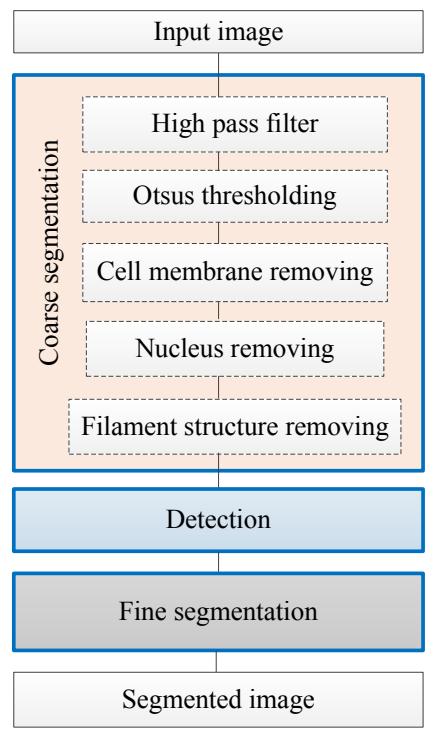

Fig. 1. Mitochondrial segmentation strategy

[19]. Furthermore, the Gabor feature is convinced to be welladapted to fiber structure extraction which was contained in variety applications such as fingerprint classification [21], Iris recognition [22], prostate cancer image segmentation and recognition [23], blood vessel detection [20]. This effectiveness can be explained by the property of both frequency and orientation selective which provides a joint optimal resolution in both frequency and spatial domain. As a result, we applied the Gabor filter for extracting features of ridge and valley or fiber structures which include in mitochondria image texture and does not appear on other big organelles.

We utilize the maximum magnitude of Gabor coefficient to represent for a region in detection task. The magnitude of Gabor coefficient at a pixel is computed as follows:

$$
G(X, Y)=\left|\sum_{x=-\frac{w}{2}}^{\frac{w}{2}} \sum_{y=-\frac{w}{2}}^{\frac{w}{2}} I(X+x, Y+y) g_{\lambda, \theta, \gamma}(x, y)\right|
$$

where $I$ is a window of size $w \times w$ and $g$ is the Gabor filter defined by [18]:

$$
g_{\lambda, \theta, \gamma}(x, y)=\exp \left(-\frac{x_{\theta}^{2}+\gamma y_{\theta}^{2}}{2 \sigma^{2}}\right) \exp \left(j \frac{2 \pi}{\lambda} x_{\theta}\right)
$$

where $x_{\theta}=x \cos \theta+y \sin \theta$ and $y_{\theta}=-x \sin \theta+y \cos \theta, \lambda$ is the wavelength of the sinusoidal plane, $\theta$ is the orientation of the Gabor filter, $\sigma$ is the standard deviation of $x$-axis, and $\gamma$ is the ratio of the standard deviation of $x$-axis and the standard deviation of $y$-axis.

\section{Step 3: Fine segmentation}

The coarse segmented regions in Step 1 which have been decided to include a mitochondria through Step 2 will be the input for a fine segmentation operator. Since this segmented region is small, the mitochondria fine segmentation is not a 


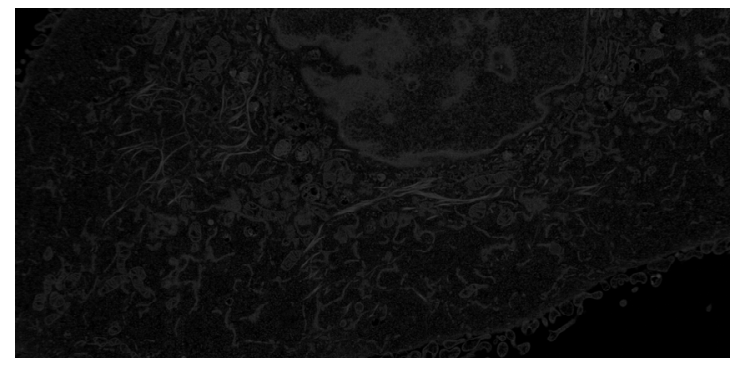

Fig. 2. FIB-SEM image of intracellular space

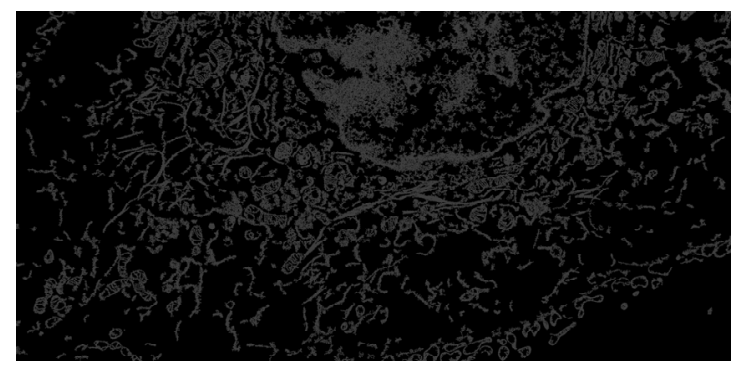

Fig. 3. Binary image of intracellular space

big task. A similar combination of morphology operators and Canny edge detection method as used in Step 1 is adopted for this step.

\section{EXPERIMENT}

The FIB-SEM intracellular images, which were collected from the cancer cell line derived from a human head and neck squamous cell carcinoma (SCC-61) parental line [14], [15], were used in this study.

Figure 2 shows the FIB-SEM scan of the intracellular space of the cancer cell SCC-61. It can be easy to recognize that the structure of this cell is very complicated which causes a difficulty for segmentation and analysis. Figures 3 is the first initial result which is obtained after high pass filtering, thresholding and small objects removing. After a further removing cell membrane step, nucleus and filament structures through a coarse segmentation strategy described in section II, the result image is shown in Figure 4. At this point, the suspected regions that contain mitochondria and similar mitochondria shape areas are isolated by such the coarse segmentation process.

Because of strong correlated properties between the mitochondria image texture and the Gabor filters, the Gabor coefficients at mitochondria areas are higher than the other parts as shown in Figure 5. The parameters designed for the Gabor filters derive from spectrum analysis of some sample mitochondria regions. The space wavelength $\lambda$ of the mitochondria is specified between the range of 4 to 5 pixels, and we select four orientation values of $\theta$ as 0,45 , 90 and 135 degrees to cover all the wide range of orientations of the ridge and valley structures of the mitochondria. The directional standard deviation ratio $\gamma$ are selected as 0.5 . A simple thresholding process applied for maximum Gabor

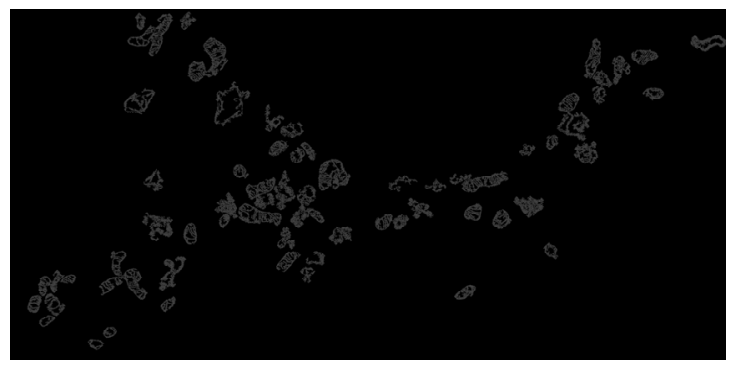

Fig. 4. Coarse segmentation of mitochondria

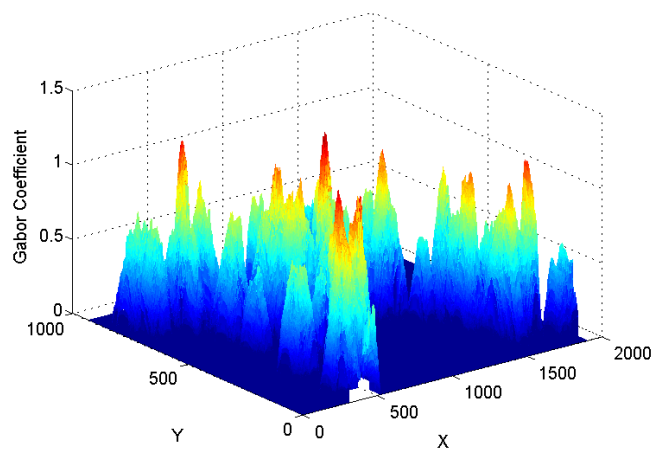

Fig. 5. Gabor Coefficients of the coarse segmentation image

coefficient corresponding to every suspected region can help to distinguish between mitochondria (Mt) and non-mitochondria $(\mathrm{X})$ regions as presented in Figure 6.

Next, in order to confirm the effectiveness of Gabor filter for mitochondrial detection, we compare the mitochondrial detection ability of our proposed regional maximum Gabor filter coefficient with other textural features including homogeneity, contrast, correlation and entropy derived from famous gray level co-occurrence matrix (GLCM). In order to present the effectiveness of detection parameters, we utilize receiver operating characteristic (ROC) curves, which is the plot of the true positive rate (TPR) and the false positive rate (FPR). The results is presented in Fig. 7. Obviously, our proposed features of maximum and standard deviation values of coarse segmented regions outperforms the textural features extracted based on GLCM for mitochondria detection.

Detected regions provide small and isolated areas which facilitate the fine mitochondrial segmentation. Two of such isolated area samples are shown in the middle row of Figure 8. The corresponding cropped regions from original image are shown in the top row. The fine segmentation of these mitochondria containing regions are shown in the bottom row of the Figure 8. It can be obviously seen that the segmentation results can be largely improved by this fine segmentation step in comparison to those given by the coarse segmentation step.

\section{CONCLUSIONS}

We have presented a simple three-stage approach for mitochondrial segmentation. The proposed method utilizes the exclusive property of image segmentation and the effective feature extraction of Gabor filter for segmenting mitochondria. 


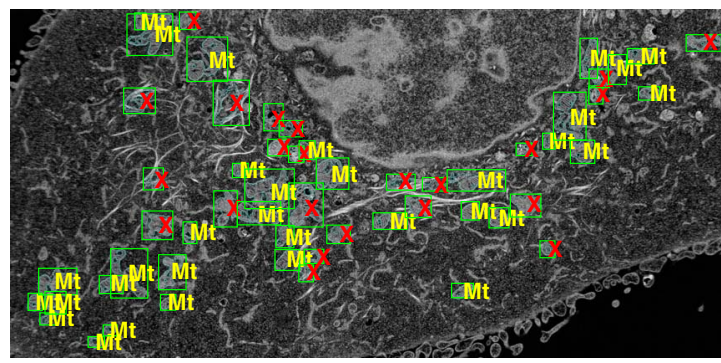

Fig. 6. Gabor filter-based detection results

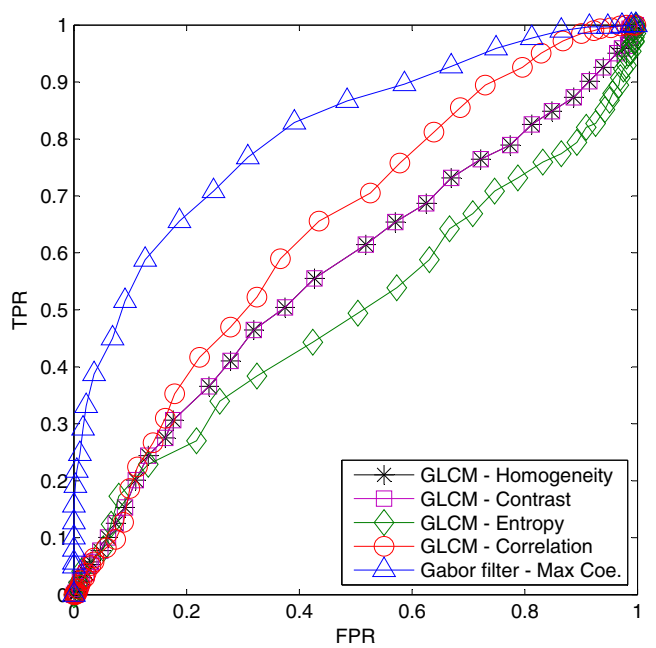

Fig. 7. ROC comparison of Gabor filter-based and GLCM-based detector

Though it is just some initial results, the proposed process illustrates an appropriately simple implementation for the hard whole intracellular image analysis problem of both cancer and normal cellular.

\section{ACKNOWLEDGMENTS}

We thank Dr. Daisuke Hoshino and Professor Motoharu Seiki of Division of Cancer Cell Research, Institute of Medical Science, University of Tokyo, for providing cancer cell images.

\section{REFERENCES}

[1] G Kroemer, "Mitochondria in cancer" , Oncogene, Vol. 25, pp. 46304632,2006

[2] Gogvadze V, Orrenius S, and Zhivotovsky B.,“ Mitochondria in cancer cells: what is so special about them?", Trends Cell Biol., 18(4):165-73., 2008.

[3] Douglas C. Wallace, "Mitochondria and cancer", Nature Reviews Cancer , Vol. 12, pp. 685-698, 2012.

[4] Simone Fulda, Lorenzo Galluzzi and Guido Kroemer, "Targeting mitochondria for cancer therapy", Nature Reviews Drug Discovery, Vol. 9, pp. 447-464, 2010.

[5] Wasilewski M. and Scorrano L., "The changing shape of mitochondrial apoptosis", Trends Endocrinol Metab, Vol. 20(6), pp.87-94, 2009.

[6] G. Melino, M. Karbowski, and R. J. Youle, "Dynamics of mitochondrial morphology in healthy cells and during apoptosis", Cell Death and Differentiation, Vol. 10, pp.870-880, 2003.

[7] Campello S. and Scorrano L., "Mitochondrial shape changes: orchestrating cell pathophysiology", EMBO Rep., Vol.11(9), pp.678-84, 2010.
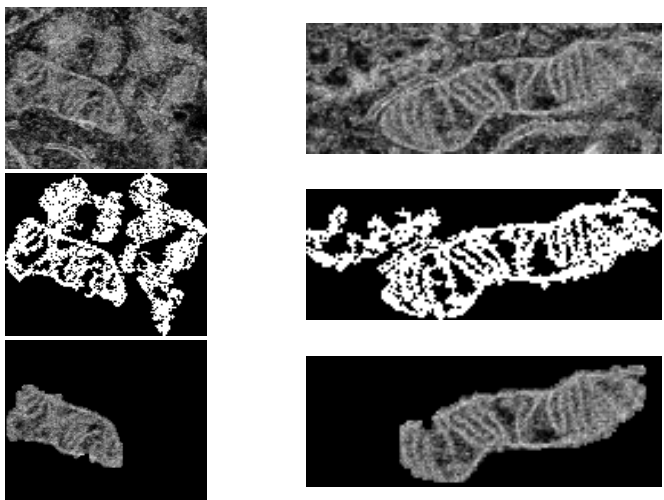

Fig. 8. Fine segmentation of mitochondria

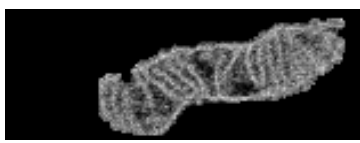

[8] Sitansu Kumar Das, Sanjoy Kumar Saha, Dipti Prasad Mukherjee, "Segmentation of multiple objects evolving conditional random field based topology adaptive active membrane," Signal Processing, Vol. 92, p.p. 2341-2355, 2012.

[9] Bjoern Andres, Ullrich Koethe, Thorben Kroeger, Moritz Helmstaedter, Kevin L. Briggman, Winfried Denk, Fred A. Hamprecht, "3D segmentation of SBF-SEM images of neuropil by a graphical model over supervoxel boundaries," Medical Image Analysis, Vol. 16, p.p. 796-805, 2012.

[10] A. Lucchi, K. Smith, R. Achanta, G. Knott, P. Fua, "Supervoxel-Based Segmentation of Mitochondria in EM Image Stacks With Learned Shape Features," Medical Imaging, IEEE Transactions on , vol.31, no.2, pp.474486, 2012.

[11] Rajesh Narasimha, Hua Ouyang, Alexander Gray, Steven W. McLaughlin, Sriram Subramaniam, "Automatic joint classification and segmentation of whole cell 3D images," Pattern Recognition, Vol. 42, p.p. 10671079, 2009.

[12] Vitaladevuni SN, Sofroniew NJ, Mishchenko Y, Genkin A, Chklovskii DB, Harris K, "Automatic mitochondria detection in electron micrographs," MIAAB, 2008.

[13] D.J. Stokes, F. Morrissey, B.H. Lich, "A new approach to studying biological and soft materials using focused ion beam scanning electron microscopy (FIB SEM)," Journal of Physics: Conference Series, Vol.26, pp. 50-53, 2006.

[14] E.S. Clark, A.S. Whigham, W.G. Yarbrough, A.M. Weaver, "Cortactin is an essential regulator of matrix metalloproteinase secretion and extracellular matrix degradation in invadopodia," Cancer Res, Vol. 67, pp. 4227-4235, 2007.

[15] D. Hoshino et al, "Establishment and validation of computational model for MT1-MMP ependent ECM degradation and intervention strategies," PLoS Comput Biol, Vol.8, 2012.

[16] Canny, J., "A Computational Approach To Edge Detection,” IEEE Trans. Pattern Analysis and Machine Intelligence, Vol. 8(6), pp. 679-698, 1986.

[17] Nobuyuki Otsu . "A threshold selection method from gray-level histograms". IEEE Trans. Sys., Man., Cyber., Vol. 9 (1), pp. 62-66, 1979.

[18] S. E. Grigorescu, et al., "Comparison of texture features based on Gabor filters," Image Processing, IEEE Transactions on, vol. 11, pp. 1160-1167, 2002.

[19] J. K. Kamarainen, et al., "Invariance properties of Gabor filter-based features-overview and applications," Image Processing, IEEE Transactions on, vol. 15, pp. 1088-1099, 2006.

[20] D. Wu, et al., "On the adaptive detection of blood vessels in retinal images," Biomedical Engineering, IEEE Transactions on, vol. 53, pp. 341-343, 2006

[21] Y. Zhang and X. Jing, "Spectral analysis based fingerprint image enhancement algorithm," in Image Analysis and Signal Processing (IASP), 2010 International Conference on, 2010, pp. 656-659.

[22] T. Chung-Chih, et al., "Iris Recognition Using Possibilistic Fuzzy Matching on Local Features," Systems, Man, and Cybernetics, Part B: Cybernetics, IEEE Transactions on, vol. 42, pp. 150-162, 2012.

[23] S. S. Mohamed and M. M. A. Salama, "Prostate Cancer Spectral Multifeature Analysis Using TRUS Images," Medical Imaging, IEEE Transactions on, vol. 27, pp. 548-556, 2008. 